\title{
Fluorescence depolarization by anisotropic rotational diffusion of a luminophore and its carrier molecule
}

\author{
Thomas P. Burghardta) \\ Department of Physics and Biophysics Research Division, University of Michigan, Ann Arbor, Michigan 48109 \\ (Received 30 November 1982; accepted 10 February 1983)

\begin{abstract}
The analytical expression for the polarization anisotropy is derived for a luminophore undergoing rotational diffusion about a single axis while attached to a nonluminescing, rotationally diffusing, symmetrical carrier molecule. In contrast to previous related calculations, the rotation axis of the luminophore is assumed to have an arbitrary orientation relative to the carrier. Additionally, the polarization anisotropy is measured for bovine serum albumin (BSA) labeled with dansyl, NBD, rhodamine, or eosin that is: (a) surface adsorbed to a glass/buffer interface, using a variation of the technique of total internal reflection fluorescence spectroscopy (TIRFS), or (b) bulk dissolved, using conventional transmitted illumination fluorescence spectroscopy. With this theory, using previously published values for the rotational diffusion constants of BSA and the fluorescence lifetimes of the fluorophores, the rotational diffusion constant of the covalently bound probes is estimated from the measured anisotropy values. The results indicate a wide variability in the rotational diffusion constant of the probes (from $\sim 10^{7} \mathrm{~s}^{-1}$ for dansyl to $\sim 10^{9} \mathrm{~s}^{-1}$ for eosin) attached to both the surface adsorbed and bulk dissolved forms of BSA. Contrasting the rotational diffusion constant for each probe for surface adsorbed BSA vs bulk dissolved BSA indicates surface adsorption of the BSA molecule inhibits the rotational motion of the probe. These results have important implications in the application of other fluorescence techniques, such as singlet-singlet energy transfer, where the rotational mobility of the probe is important.
\end{abstract}

\section{INTRODUCTION}

The polarization of emitted light from a luminophore undergoing rotational diffusion, after polarized excitation, can be used to study the shape of the luminophore and properties of the local medium surrounding the luminophore. Fluorescence and phosphorescence depolarization after polarized excitation are useful techniques for studying the rotational diffusion of proteins under a variety of conditions in the nanosecond to millisecond time range. These techniques have been used to determine, e.g., the shape, dimensions, and flexibility of rotationally diffusing protein molecules in solution, ${ }^{1-6}$ the internal motion of macromolecules, ${ }^{3-6}$ and the rotational mobilities of molecules embedded in a membrane. ${ }^{7-15}$

In the case of large biomolecules such as protein, depolarization of emitted light after polarized excitation occurs because of the rotational motion of the biomolecule as a rigid body as well as local rotational motion in the neighborhood of the luminophore. There can be ambiguity in the assignment of the contribution to the depolarization of emitted light from these two distinct motions. The theory of emission depolarization by rotational diffusion of a luminophore attached to a larger rotationally diffusing molecule (the carrier) has been presented earlier for spherical carrier $\mathrm{s}^{16}$ and for symmetrical carriers in the special case where the luminophore is so oriented that its motion does not rotate its principal frame (the frame in which its diffusion tensor is diagonal) from the principal frame of the carrier. ${ }^{7,17}$ This paper outlines a derivation of the polarization anisotropy for a rotationally diffusing luminophore attached to a rotationally diffusing asymmetrical carrier.

\footnotetext{
2)Present address: Cardiovascular Research Institute, University of California, San Francisco, California 94143.
}

Applying this theory to bulk dissolved fluorescently labeled bovine serum albumin (BSA) and, given known rotational diffusion constants of BSA, ${ }^{5,18}$ the local rotational diffusion constant is estimated for the covalently attached fluorescent groups: dansyl, NBD, eosin, and rhodamine.

Additionally, the technique of emission depolarization after polarized excitation is applied to biomolecules adsorbed at a solid/liquid interface using a totally internally reflected, polarized excitation source. With the technique of polarized, total internal reflection fluorescence (TIRF), fluorescence depolarization studies on molecules adsorbed at a solid/liquid interface is possible while the adsorbed molecules are in chemical equilibrium with the bulk dissolved ones. Here experimental results are presented of depolarization studies of fluorescent labeled BSA adsorbed to a quartz/buffer interface. [Polarized TIRF is a particular example of a general method of total internal reflection fluorescence spectroscopy (TIRFS) described in a separate paper. $\left.{ }^{19}\right]$

A wide variability is observed in the local rotational diffusion constants among the various fluorescent probes studied, for the carrier in either the bulk dissolved or surface adsorbed form. Comparison of the diffusion constants of the probes for the carrier in each form indicates surface adsorption of the carrier causes inhibition in the local rotational mobility of the fluorescent group.

\section{THEORY}

\section{The equation of motion}

The polarization anisotropy of a rotationally diffusing rigid body has been derived. ${ }^{1,20}$ The anisotropy is derived here for the more complicated system of a small 
rotationally diffusing luminophore attached to a rotationally diffusing rigid carrier molecule.

Let subscript " 1 " pertain to the luminophore and " 2 " to the carrier molecule. The Euler angles $\alpha_{2}, \beta_{2}, \gamma_{2}$ and $\alpha_{1}, \beta_{1}, \gamma_{1}$ rotate the lab frame into the principal frame of the carrier and from there to the principal trame of the luminophore, respectively. Abbreviating $\alpha_{1}, \beta_{1}, \gamma_{1}$ with $\Omega_{1}$ and $\alpha_{2}, \beta_{2}, \gamma_{2}$ with $\Omega_{2}$, we define $P\left(\Omega_{1}\right.$, $\left.\Omega_{2}, t\right) d \Omega_{1} d \Omega_{2}$ as the probability that a luminophore and its carrier have orientations within $d \Omega_{1}$ and $d \Omega_{2}$ about $\Omega_{1}$ and $\Omega_{2}$, respectively. The luminophore rotationally diffuses because of both rotational motion of the carrier and local rotational motion relative to the carrier. The rotational diffusion equation for this system is

$$
\begin{aligned}
\frac{\partial}{\partial t} P\left(\Omega_{1}, \Omega_{2}, t\right)= & \left(\sum_{i=1}^{3} d_{i, i} S_{i}^{2}+\sum_{i=1}^{3} \sum_{j=1}^{3} D_{i, j} L_{i} L_{j}\right) \\
& \times P\left(\Omega_{1}, \Omega_{2}, t\right),
\end{aligned}
$$

where $d_{i, i}$ is the rotational diffusion tensor and $S_{i}$ the $i$ th component of the orbital angular momentum operator ${ }^{20,21}$ of the luminophore in its principal frame; $D_{i, j}$ is the rotational diffusion tensor and $L_{i}$ the $i$ th component of the orbital angular momentum operator of the carrier molecule in the principal frame of the luminophore. Equation (1) can be rewritten, after expressing the tensors $D_{i, j}$ and $L_{i} L_{j}$ in a spherical basis ${ }^{21}$ as

$$
\begin{aligned}
\frac{\partial}{\partial t} P\left(\Omega_{1}, \Omega_{2}, t\right)= & \left(\sum_{i=1}^{3} d_{i, i} S_{i}^{2}\right. \\
& \left.+\frac{1}{3} D L^{2}+\sum_{m=-2}^{2}(-1)^{m} N_{2,-m} T_{2, m}\right) P\left(\Omega_{1}, \Omega_{2}, t\right)
\end{aligned}
$$

where $D=D_{1,1}+D_{2,2}+D_{3,3}$, and $N_{2}, T_{2}$ are spherical tensors. $N_{2}$ and $T_{2}$ are given by ${ }^{21}$

$$
\begin{aligned}
& N_{2, m}=\sum_{m^{\circ}=-2}^{2} D_{m^{*}, m}^{2}\left(\Omega_{1}\right) D_{2, m^{\circ}}^{\phi f}, T_{2,0}=\frac{1}{\sqrt{6}}\left(L^{2}-3 L_{3}^{2}\right), \\
& T_{2, \pm 1}=-\frac{1}{\sqrt{2}} L_{\star}\left(2 L_{3} \mp 1\right), T_{2, \pm 2}=-L_{ \pm}^{2},
\end{aligned}
$$

where $D_{m^{\prime}, m}^{2}\left(\Omega_{1}\right)$ is the Wigner rotation matrix for the rotation from the principal frame of the carrier to the principal frame of the luminophore. $D_{2, m}^{p f}$ is the spherical rotational diffusion tensor of the carrier in its principal frame and $L_{t}=\mp\left(L_{1} \pm i L_{2}\right) / \sqrt{2}$. Elements of $D_{2, m^{\prime}}^{f f}$ are constants and given in terms of $D_{i, j} .{ }^{21}$

Constraining the luminophore motion to one degree of rotational freedom (about the luminophore principle frame $z$ axis) and separating the time dependence by the separation of variables method, we find

$$
E p\left(\Omega_{1}^{0}, \Omega_{2}\right)=\mathbf{E} p\left(\Omega_{1}^{0}, \Omega_{2}\right),
$$

where $E$ is a constant, $p$ is the angular dependent part of $P$, and operator $\mathbf{E}$ is

$$
\mathbf{E}=d_{3,3} S_{3}^{2}+\frac{1}{3} D L^{2}+\sum_{m=-2}^{2}(-1)^{m} N_{2,-m} T_{2, m} .
$$

The argument $\Omega_{1}^{0}$ designates the angles $\alpha_{1}^{0}, \beta_{1}^{0}, \gamma_{1}$ with $\alpha_{1}^{0}$ and $\beta_{1}^{0}$ constant in time (because the luminophore motion is constrained) and $\gamma_{1}$ time dependent. The eigenvector solution to $\mathrm{Eq} .(4),|l, \tau\rangle$, is a linear combination of the product of basis vectors $\{l, \lambda\rangle$ and $|k\rangle$, so that

$$
|l, \tau\rangle=\sum_{\lambda, k} a_{\lambda, k}^{\tau}|l, \lambda\rangle|k\rangle \equiv \sum_{\lambda, k} a_{\lambda, k}^{\tau}|l, \lambda, k\rangle,
$$

where $|l, \lambda\rangle$ are symmetric top eigenvectors for the carrier molecule, ${ }^{22}$ given by

$$
\left\langle\Omega_{2} \mid l, \lambda\right\rangle=(-1)^{v}\left(\frac{2 l+1}{8 \pi^{2}}\right)^{1 / 2} D_{-v,-\lambda}^{l}\left(\Omega_{2}\right),
$$

$|k\rangle$ are eigenvectors for the luminophore, given by

$$
\left\langle\gamma_{1} \mid k\right\rangle=\frac{1}{\sqrt{2 \pi}} \exp \left(-i k \gamma_{1}\right),
$$

and $a_{\lambda, k}^{\top}$ are constants. Using Eqs. (3) and (5), and the equations below ${ }^{22}$ :

$$
\begin{aligned}
& L^{2}|l, \lambda\rangle=l(l+1)|l, \lambda\rangle, \quad L_{3}|l, \lambda\rangle=\lambda|l, \lambda\rangle, \\
& L_{ \pm}|l, \lambda\rangle=\mp\left[\frac{1}{2}(l \pm \lambda)(l \mp \lambda+1)\right]^{1 / 2}|l, \lambda \mp 1\rangle, \\
& S_{3}|k\rangle=k|k\rangle, \quad\left\langle k^{\prime} \mid k\right\rangle=\delta_{k^{\prime}, k},\left\langle\lambda^{\prime}, l^{\prime} \mid l, \lambda\right\rangle=\delta_{l^{\prime}, l^{\prime} \delta_{\lambda^{\prime}, \lambda},},
\end{aligned}
$$

where $\delta_{i, j}$ is the Kronecker delta, it is possible to show

$$
\begin{aligned}
\left\langle k^{\prime}, \lambda^{\prime}, l|\mathbf{E}| l, \lambda, k\right\rangle= & \left\{d_{3,3} k^{2}+\frac{1}{3} D l(l+1)+\frac{1}{\sqrt{6}} g_{0}\left[l(l+1)-3 \lambda^{2}\right]\right\} \delta_{\lambda^{\prime}, \lambda} \delta_{k^{\prime}, k}-\frac{1}{2} g_{2}\left[[(l-\lambda)(l-\lambda-1)(l+\lambda+2)(l+\lambda+1)]^{1 / 2}\right. \\
& \left.\times \delta_{\lambda^{\prime}, \lambda+2} \delta_{k^{\prime}, k-2}\right\}-\frac{1}{2} g_{2}^{*}\left\{[(l+\lambda)(l+\lambda-1)(l-\lambda+2)(l-\lambda+1)]^{1 / 2} \delta_{\lambda^{\prime}, \lambda-2} \delta_{k^{\prime}, k+2}\right\} \\
& +\frac{1}{2} g_{1}\left\{(2 \lambda+1)[(l+\lambda+1)(l-\lambda)]^{1 / 2} \delta_{\lambda^{\prime}, \lambda+1} \delta_{k^{\prime}, k-1}\right\}+\frac{1}{2} g_{1}^{*}\left\{(2 \lambda-1)[(l-\lambda+1)(l+\lambda)]^{1 / 2} \delta_{\lambda^{\prime}, \lambda-1} \delta_{k^{\prime}, k+1},\right.
\end{aligned}
$$

where

$$
g_{m} \equiv D_{2,2}^{p f}\left[\exp \left(-2 i \alpha_{1}^{0}\right) d_{-2, m}^{2}\left(\beta_{1}^{0}\right)+\exp \left(2 i \alpha_{1}^{0}\right) d_{2, m}^{2}\left(\beta_{1}^{0}\right)\right]+D_{2,0}^{p f} d_{0, m}^{2}\left(\beta_{1}^{0}\right)
$$

and $d_{m, n}^{l}\left(\beta_{1}^{0}\right)$ is a reduced Wigner rotation matrix.

\section{The polarization anisotropy}

The polarization anisotropy is defined by

$$
r(t) \equiv \frac{I_{11}-I_{2}}{I_{n}+2 I_{1}},
$$

where $I$ is the intensity of the emitted light, with $\|$ and $\perp$ polarization to the excitation beam polarization. For a luminophore with unit absorption and emission dipoles $\hat{\mu}_{a}$ and $\hat{\mu}_{e}$, the probability of excitation by light polarized in 
the $\hat{z}$ direction of the fixed lab frame is proportional to $\left(\hat{z} \cdot \hat{\mu}_{a}\right)^{2}$. Emitted light intensity with polarization $\hat{z}(\hat{x})$ in the lab frame is proportional to $\left[\hat{z}(\hat{x}) \cdot \hat{\mu}_{e}\right]^{2}$. Defining the quantities

$$
G_{1} \equiv\left(\hat{z} \cdot \hat{\mu}_{a}\right)^{2}, \quad G_{2} \equiv\left(\hat{z} \cdot \hat{\mu}_{e}\right)^{2}-\left(\hat{x} \cdot \hat{\mu}_{\varepsilon}\right)^{2}, \quad G_{3} \equiv\left(\hat{z} \cdot \hat{\mu}_{e}\right)^{2}+2\left(\hat{x} \cdot \hat{\mu}_{e}\right)^{2},
$$

the probability distribution $P\left(\Omega_{1}^{0}, \Omega_{2}, t\right)$ has the form

$$
P\left(\Omega_{1}^{0}, \Omega_{2}, t\right)=\frac{1}{\left\langle G_{1}\right\rangle} \sum_{T, l}\left\langle\tau, l \mid G_{1}\right\rangle\left\langle\Omega_{1}^{0}, \Omega_{2} \mid l, \tau\right\rangle \exp \left(-E_{l, \tau} t\right)
$$

and the anisotropy is

$$
r(t)=\frac{\left\langle G_{2}^{*} \mid P\right\rangle}{\left\langle G_{3}^{*} \mid P\right\rangle}
$$

Spherical vectors in the luminophore frame are related to the lab frame with a Wigner rotation matrix. This matrix can be written as a product of two matrices corresponding to two successive rotations, from the lab frame to the principal frame of the carrier and then to the principal frame of the luminophore. This implies Eq. (13) can be written in the form

$$
\begin{aligned}
& G_{1}=\sum_{q q^{\prime}} \sum_{n n^{\prime}}(-1)^{\alpha+q^{*}} D_{n, a}^{1}\left(\Omega_{1}^{0}\right) D_{n^{\prime}, q^{\prime}}^{1}\left(\Omega_{1}^{0}\right) D_{0, n}^{1}\left(\Omega_{2}\right) D_{0, n^{\prime}}^{1}\left(\Omega_{2}\right) \mu_{a}^{-\alpha} \mu_{a}^{-\alpha^{*}},
\end{aligned}
$$

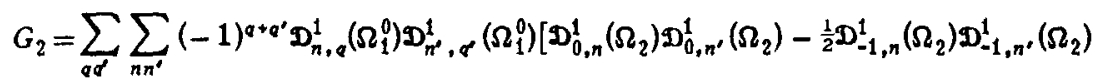

$$
\begin{aligned}
& \left.-\frac{1}{2} \mathscr{D}_{1, n}^{1}\left(\Omega_{2}\right) D_{1, n^{\prime}}^{1}\left(\Omega_{2}\right)+\frac{1}{2} D_{1, n}^{1}\left(\Omega_{2}\right) D_{-1, n^{\prime}}^{1}\left(\Omega_{2}\right)+\frac{1}{2} \mathcal{D}_{-1, n}^{1}\left(\Omega_{2}\right) D_{1, n^{\prime}}^{1}\left(\Omega_{2}\right)\right] \mu_{e}^{-q} \mu_{e}^{-a^{\prime}}, \\
& G_{3}=\sum_{q q^{\prime}} \sum_{n n^{\prime}}(-1)^{q+\alpha^{\prime}} D_{n_{0}}^{1}\left(\Omega_{1}^{0}\right) D_{n^{\prime}, q^{\prime}}^{1}\left(\Omega_{1}^{0}\right)\left[D_{0, n}^{1}\left(\Omega_{2}\right) D_{0, n^{\prime}}^{1}\left(\Omega_{2}\right)+D_{-1, n}^{1}\left(\Omega_{2}\right) D_{-1, n^{\prime}}^{1}\left(\Omega_{2}\right)\right. \\
& +D_{1, n}^{1}\left(\Omega_{2}\right) D_{1, n^{\prime}}^{1}\left(\Omega_{2}\right)-D_{1, n}^{1}\left(\Omega_{2}\right) D_{-1, n^{\prime}}^{1}\left(\Omega_{2}\right)-D_{-1, n}^{1}\left(\Omega_{2}\right) D_{1, n}^{1}\left(\Omega_{2}\right) \mu_{e}^{-q} \mu_{e}^{-\alpha^{\prime}} .
\end{aligned}
$$

Calculation of $r(t)$ is performed using Eqs. (7), (8), and (14)-(16) and the following relations $\mathbf{s}^{22}$ :

$$
\begin{aligned}
& \int d \Omega D_{\mu_{1}, m_{1}}^{j_{1}^{*}}(\Omega) D_{\mu_{2}, m_{2}}^{j_{2}}(\Omega)=\frac{8 \pi^{2}}{2 j_{1}+1} \delta_{\mu_{1}, \mu_{2}} \delta_{m_{1}, m_{2}} \delta_{j_{1}, j_{2}}, \\
& \int d \Omega D_{\mu_{3}, m_{3}}^{j_{3}^{*}}(\Omega) D_{\mu_{2}, m_{2}}^{j_{2}}(\Omega) D_{\mu_{1}, m_{1}}^{j_{1}}(\Omega)=\frac{8 \pi^{2}}{2 j_{3}+1} \delta_{\mu_{1}+\mu_{2}, \mu_{3}} \delta_{m_{1} m_{2}, m_{3}}\left\langle j_{1}, j_{2}, \mu_{1}, \mu_{2} \mid j_{3}, \mu_{3}\right\rangle\left\langle j_{1}, j_{2}, m_{1}, m_{2} \mid j_{3}, m_{3}\right\rangle, \\
& D_{m^{\prime}, m^{\prime}}^{j^{*}}(\Omega)=(-1)^{m^{\prime}-m_{1}} D_{-m^{\prime},-m}^{j}(\Omega), d_{m_{1}, k_{1}}^{1}(\beta) d_{m_{2}, k_{2}}^{1}(\beta)=\sum_{j}\left\langle 1,1, m_{1}, m_{2} \mid j, m\right\rangle d_{m, k}^{j}(\beta)\left\langle 1,1, k_{1}, k_{2} \mid j, k\right\rangle,
\end{aligned}
$$

where $\left\langle j_{1}, j_{2}, m_{1}, m_{2} \mid j_{3}, m_{3}\right\rangle$ are Clebsch Gordan coefficients. ${ }^{22}$ One finds

$$
\begin{aligned}
r(t)= & \frac{3}{5} \sum_{\tau} \sum_{a q^{\prime}} \sum_{k k^{\prime}}(-1)^{k} \sum_{\lambda}\left\{(-1)^{\lambda} a_{\lambda, k}^{* \tau} \exp \left(-i \lambda \alpha_{1}^{0}\right) d_{\lambda, k}^{2}\left(\beta_{1}^{0}\right)\right\} \sum_{\lambda^{\prime}}\left\{(-1)^{\lambda^{\prime}} a_{\lambda^{\prime}, k^{\prime}}\right. \\
& \left.\times \exp \left(i \lambda^{\prime} \alpha_{1}^{0}\right) d_{\lambda^{\prime}, k^{\prime}}^{2}\left(\beta_{1}^{0}\right)\right\}\left\langle 1,1, q^{\prime}, k^{\prime}-q^{\prime} \mid 2, k^{\prime}\right\rangle\langle 1,1, q,-k-q \mid 2,-k\rangle \mu_{a}^{-\alpha} \mu_{a}^{k+\mu_{a}} \mu_{e}^{-\alpha} \mu_{e}^{\sigma^{--k^{\prime}}} \exp \left(-E_{2, \tau^{\prime}} t\right) .
\end{aligned}
$$

Defining $b_{k}^{\top}$ with the expression $a_{\lambda, k}^{\top}=(-1)^{\lambda} b_{k}^{\tau} d_{\lambda, k}^{\tau}\left(\beta_{1}^{0}\right)$ and using the identity

$$
\sum_{\lambda} d_{\lambda, k}^{2}(\beta) d_{\lambda, k^{\prime}}^{2}(\beta)=\delta_{k, k^{\prime}}
$$

it is possible to show

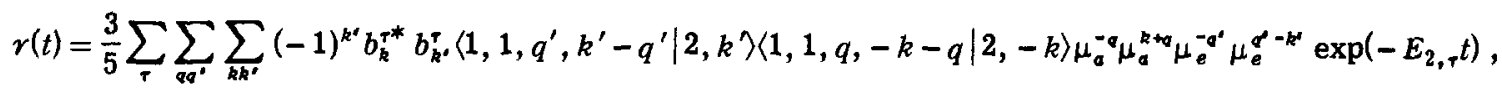

where $E_{2, \uparrow}$ and $b_{k}^{t}$ are calculated when diagonalizing the matrix $\mathbf{M}$ such that

$$
\mathbf{M}_{k^{\prime}, k}=\sum_{\lambda^{\prime} \lambda}(-1)^{\lambda^{\prime}+\lambda} \exp \left[i \alpha_{1}^{0}\left(\lambda^{\prime}-\lambda\right)\right] d_{\lambda^{\prime}, k^{\prime}}^{2}\left(\beta_{1}^{0}\right) d_{\lambda, k}^{2}\left(\beta_{1}^{0}\right)\left\langle k^{\prime}, \lambda^{\prime}, 2|\mathbf{E}| 2, \lambda, k\right\rangle \text {. }
$$

Diagonalization of $\mathbf{M}$, in general, requires finding the roots of a fifth order polynomial. This is possible numerically and is not pursued further here (the special cases of $\beta_{1}^{0}=0, \pi / 2$ can be handled analytically by the method described below). For a symmetrical carrier, where the spherical diffusion tensor element $D_{2,2}^{\phi f}=0$, it can be shown that eigenvectors of $\mathbf{M}$, in terms of the basis vectors $|k\rangle$, are

$$
|l, \tau\rangle=\left\{\begin{array}{l}
c_{0}^{\top}|0\rangle+\frac{c_{1}^{\top}}{\sqrt{2}}\left[\exp \left(-i \alpha_{1}^{0}\right)|1\rangle-\exp \left(i \alpha_{1}^{0}\right)|-1\rangle\right]+\frac{c_{2}^{\top}}{\sqrt{2}}\left[\exp \left(-2 i \alpha_{1}^{0}\right)|2\rangle+\exp \left(2 i \alpha_{1}^{0}\right)|-2\rangle\right] ; \tau=1,2,3 \\
\frac{c_{1}^{\top}}{\sqrt{2}}\left[\exp \left(-i \alpha_{1}^{0}\right)|1\rangle+\exp \left(i \alpha_{1}^{0}\right)|-1\rangle\right]+\frac{c_{2}^{\tau}}{\sqrt{2}}\left[\exp \left(-2 i \alpha_{1}^{0}\right)|2\rangle-\exp \left(2 i \alpha_{1}^{0}\right)|-2\rangle\right] ; \tau=4,5,
\end{array}\right.
$$


where $c_{1}^{\tau}$ are real constants. With these new orthonormal basis vectors, calculation of $E_{2, \tau}, c_{i}^{\top}$, and $b_{k}^{\top}$ is performed when diagonalizing a $3 \times 3$ and a $2 \times 2$ matrix ( $Q$ for $\tau=1,2,3$ and $\mathbf{R}$ for $\tau=4,5$ ) independently. This calculation is outlined in the Appendix.

The values for $b_{k}^{\top}$ in the Appendix are used in Eq. (19) to give

$$
r(t)=\frac{3}{5} \sum_{\tau=1}^{5} \sum_{m=0}^{2} \sum_{n=0}^{m} H_{n, m} c_{n}^{r} c_{m}^{\tau} \exp \left(-E_{2, \tau} t\right),
$$

where, in the notation $\hat{\mu}_{e}=\left(x_{e}, y_{e}, z_{e}\right)$ and $\hat{\mu}_{a}=\left(x_{a}, y_{a}, z_{a}\right)$ one has

$$
\begin{aligned}
& H_{0,0} \equiv 1 / 6\left(3 z_{e}^{2}-1\right)\left(3 z_{a}^{2}-1\right), \\
& H_{1,1} \equiv Z_{e} Z_{a}\left\{x_{e} x_{a}\left(1+\cos 2 \alpha_{1}^{0}\right)+y_{e} y_{a}\left(1-\cos 2 \alpha_{1}^{0}\right)-\left(x_{e} y_{a}+x_{a} y_{e}\right) \sin 2 \alpha_{1}^{0}\right\}, \\
& H_{2,2} \equiv 1 / 4\left\{\left(x_{e}^{2}-y_{e}^{2}\right)\left(x_{a}^{2}-y_{a}^{2}\right)\left(1+\cos 4 \alpha_{1}^{0}\right)+4 x_{e} y_{e} x_{a} y_{a}\left(1-\cos 4 \alpha_{1}^{0}\right)-2\left(x_{a} y_{a}\left[x_{e}^{2}-y_{e}^{2}\right]+x_{e} y_{e}\left[x_{a}^{2}-y_{a}^{2}\right] \sin 4 \alpha_{1}^{0}\right\},\right. \\
& H_{1,0} \equiv-1 / \sqrt{3}\left\{z_{e}\left(3 z_{a}^{2}-1\right)\left(x_{e} \cos \alpha_{1}^{0}-y_{e} \sin \alpha_{1}^{0}\right)+z_{a}\left(3 z_{e}^{2}-1\right)\left(x_{a} \cos \alpha_{1}^{0}-y_{a} \sin \alpha_{1}^{0}\right)\right\}, \\
& H_{2,0} \equiv 1 / 2 \sqrt{3}\left\{\left(3 z_{a}^{2}-1\right)\left(\left[x_{e}^{2}-y_{e}^{2}\right] \cos 2 \alpha_{1}^{0}-2 x_{e} y_{e} \sin 2 \alpha_{1}^{0}\right)+\left(3 z_{e}^{2}-1\right)\left(\left[x_{a}^{2}-y_{a}^{2}\right] \cos 2 \alpha_{1}^{0}-2 x_{a} y_{a} \sin 2 \alpha_{1}^{0}\right)\right\}, \\
& H_{2,1} \equiv-\left\{z _ { e } ( x _ { a } ^ { 2 } - y _ { a } ^ { 2 } ) \left(x_{e}\left[\cos \alpha_{1}^{0}+\cos 3 \alpha_{1}^{0}\right]+y_{e}\left[\sin \alpha_{1}^{0}-\sin 3 \alpha_{1}^{0}\right]+2 z_{e} x_{a} y_{a}\left(y_{e}\left[\cos \alpha_{1}^{0}-\cos 3 \alpha_{1}^{0}\right]-x_{e}\left[\sin \alpha_{1}^{0}+\sin 3 \alpha_{1}^{0}\right]\right)\right.\right. \\
&+z_{a}\left(x_{e}^{2}-y_{e}^{2}\right)\left(x_{a}\left[\cos \alpha_{1}^{0}+\cos 3 \alpha_{1}^{0}\right]+y_{a}\left[\sin \alpha_{1}^{0}-\sin 3 \alpha_{1}^{0}\right]+2 z_{a} x_{e} y_{e}\left(y_{a}\left[\cos \alpha_{1}^{0}-\cos 3 \alpha_{1}^{0}\right]-x_{a}\left[\sin \alpha_{1}^{0}+\sin 3 \alpha_{1}^{0}\right]\right)\right\} .
\end{aligned}
$$

Values for $E_{2, \tau}$ and $c_{i}^{\tau}$ are in the Appendix.

If many identical luminophores are attached to each carrier with no directional preference in the orientation of their principal frame with respect to the carrier, then the observed anisotropy is approximated by Eq. (22) averaged over angles $\alpha_{1}^{0}$ and $\beta_{1}^{0}$. Averaging of Eq. (22) over $\alpha_{1}^{0}$ can be done by inspection, averaging over $\beta_{1}^{0}$ can be done numerically.

\section{MATERIALS AND METHODS}

\section{Polarized total internal reflection and conventional} polarized illumination

Polarized excitation of the fluorophores was performed on bulk dissolved BSA, using conventional polar- ized transmitted illumination, and on surface bound BSA, using polarized TIRFS. The technique of polarized TIRFS is described below.

Total internal reflection of a light beam incident on an interface from a medium of higher refractive index (e.g., quartz glass) to a lower refractive index (e.g. , aqueous solution) occurs if the incidence angle is greater than the critical angle for total internal reflection $\theta_{c}$ $=\sin ^{-1}\left(n_{2} / n_{1}\right)$ with $n_{2}$ and $n_{1}$ referring to lower and higher refractive indices, respectively. The incident light creates an envanescent field in the lower refractive index medium with an intensity having a characteristic depth of a fraction of a wavelength of the incident light. ${ }^{23}$

The total internal reflection apparatus, described in

TABLE I. Summary of results from fluorescence polarization measurements and application of the theory to calculated $d_{3,3}$, the rotational diffusion constant, for four fluorescent probes covalently bound to BSA. Measurement of the anisotropy $r$ was made on fluorescently labeled BSA; dissolved in bulk solution, adsorbed at a quartz/buffer interface (using polarized TIRF), and immobilized in encapsulating resin (Ref. 14), $r_{b}, r_{s}$, and $r_{i}$, respectively. An approximate theory, described in detail in the text, was applied to calculate $d_{3,3}^{\prime}$ to demonstrate the significance of the results for $d_{3,3}$. The fluorescence lifetimes of the probes $\phi$ given in the

\begin{tabular}{|c|c|c|c|c|}
\hline Probe & $\phi$ & $r$ & $d_{3,3} \times 10^{-9}$ & $d_{3,3}^{\prime} \times 10^{-9}$ \\
\hline \multirow[t]{3}{*}{ Dansyl } & $13 \mathrm{~ns}$ & $r_{b}=0.153 \pm 0.003$ & $(0.0065 \pm 0.0005) \mathrm{s}^{-1}$ & $(0.012 \pm 0.002) \mathrm{s}^{-1}$ \\
\hline & & $r_{s}=0.16 \pm 0.01$ & $(0.007 \pm 0.002) \mathrm{s}^{-1}$ & $(0.017 \pm 0.004) \mathrm{s}^{-1}$ \\
\hline & & $r_{i}=0.22 \pm 0.01$ & 0 & 0 \\
\hline \multirow[t]{3}{*}{ NBD } & $3 \mathrm{~ns}$ & $r_{b}=0.156 \pm 0.002$ & $(0.075 \pm 0.003) \mathrm{s}^{-1}$ & $(0.15 \pm 0.01) \mathrm{s}^{-1}$ \\
\hline & & $r_{s}=0.21 \pm 0.01$ & $(0.013 \pm 0.001) \mathrm{s}^{-1}$ & $(0.05 \pm 0.01) \mathrm{s}^{-1}$ \\
\hline & & $r_{i}=0.26 \pm 0.01$ & 0 & 0 \\
\hline \multirow[t]{3}{*}{ Rhodamine } & $5 \mathrm{~ns}$ & $r_{b}=0.144 \pm 0.003$ & $(0.115 \pm 0.005) \mathrm{s}^{-1}$ & $(0.26 \pm 0.02) \mathrm{s}^{-1}$ \\
\hline & & $r_{s}=0.19 \pm 0.01$ & $(0.07 \pm 0.01) \mathrm{s}^{-1}$ & $(0.12 \pm 0.03) \mathrm{s}^{-1}$ \\
\hline & & $r_{i}=0.34 \pm 0.01$ & 0 & 0 \\
\hline \multirow[t]{3}{*}{ Eosin } & $0.9 \mathrm{~ns}$ & $r_{b}=0.083 \pm 0.002$ & $(1.3 \pm 0.1) \mathrm{s}^{-1}$ & $(4.7 \pm 0.5) \mathrm{s}^{-1}$ \\
\hline & & $r_{s}=0.22 \pm 0.01$ & $(0.10 \pm 0.03) \mathrm{s}^{-1}$ & $(0.17 \pm 0.03) \mathrm{s}^{-1}$ \\
\hline & & $r_{i}=0.280 \pm 0.003$ & 0 & 0 \\
\hline
\end{tabular}
table (from Refs. 6, 29, 30, and 13 for dansyl, NBD, rhodamine, and eosin, respectively) are used in all calculations. The Cartesian rotational diffusion constants of the carrier, used in the calculations, are $D_{1,1}^{p f}=D_{2,2}^{p f}=8.3 \times 10^{5} \mathrm{~s}^{-1}, D_{3,3}^{p f}=2.0 \times 10^{6} \mathrm{~s}^{-1}$ for bulk dissolved and $D_{1,1}^{p f}$ $=D_{2,2}^{p f}=D_{3,3}^{p f}=0$ for surface adsorbed. All anisotropy measurement were taken at the excitation and emission wavelengths $\lambda_{e x}$ and $\lambda_{e m}$ listed below: dansyl, $\lambda_{e x}=350 \mathrm{~nm}, \lambda_{e m}=500 \mathrm{~nm}$; NBD, $\lambda_{e x}=475 \mathrm{~nm}, \lambda_{e m}=515 \mathrm{~nm}$; eosin, $\lambda_{e x}=510 \mathrm{~nm}, \lambda_{e m}=530 \mathrm{~nm}$; rhodamine, $\lambda_{e x}=550 \mathrm{~nm}, \lambda_{\text {em }}$ $=575 \mathrm{~nm}$. 

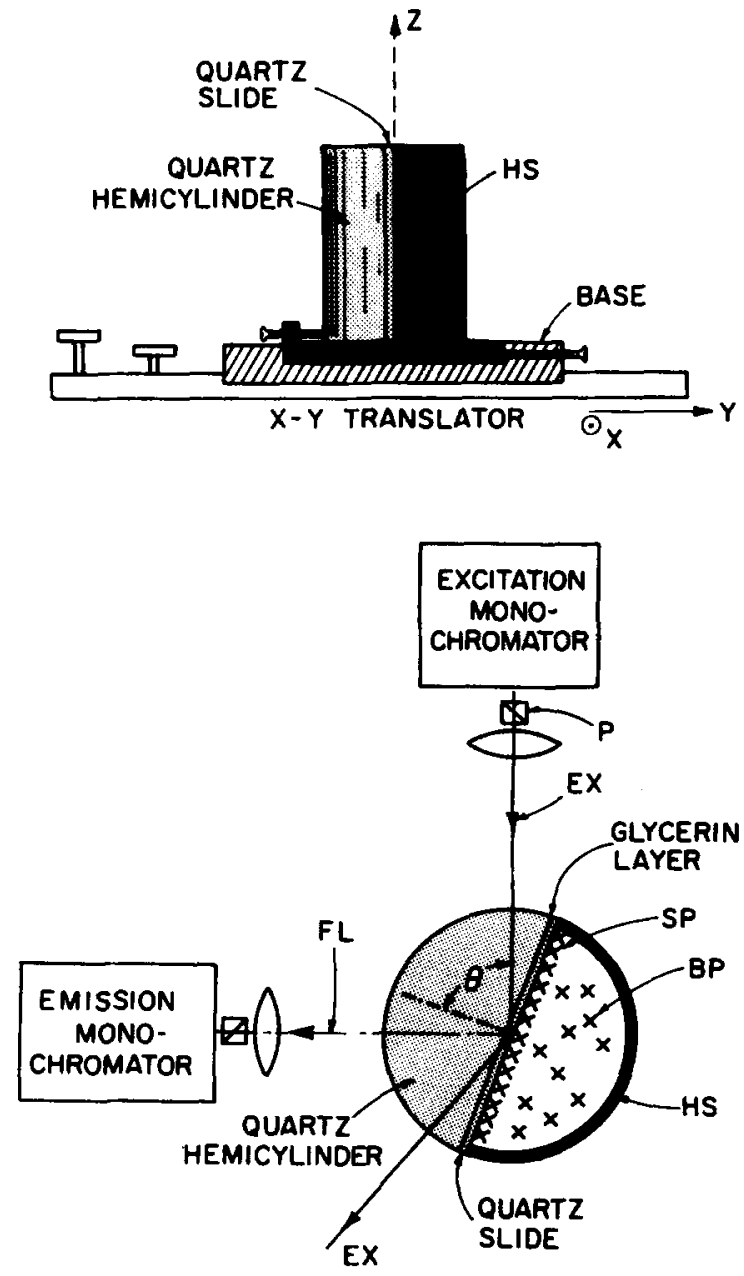

FIG. 1. Two views of the polarized total internal reflection fluorescence (TIRF) apparatus (Ref. 19). (a) Side view shows the $\hat{z}$ axis polarization of the excitation light. This polarization is necessary, given the geometry of the apparatus, to ensure the evanescent field is also linearly polarized in the $\hat{z}$ direction. The apparatus fits in the sample chamber of a commercial fluorescence spectrofluorometer. (b) The vertical view shows the paths of the excitation light (EX) and the fluorescent emission (FL) through the quartz hemicylinder. Excitation and emission light is analyzed with polarizers $(P)$. Surface adsorbed protein (SP) is illuminated by the polarized evanescent field while in chemical equilibrium with the bulk dissolved protein (BP). The angle of incidence of the excitation beam $\theta$ is $\sim 80^{\circ}$.

detail previously, ${ }^{19}$ is shown in Fig. 1. The device fits in the sample chamber of a commercial fluorescence spectrometer and fluorescence spectra on the adsorbed layer are measured in the usual manner. In the experiments, fluorescent labeled BSA molecules adsorb in equilibrium at a quartz glass/aqueous buffer interface. Only the fluorescent labeled molecules within the evanescent field become excited and fluoresce. In general, some nonadsorbed fluorescent molecules in the bulk solution within the depth of the evanescent field may be excited. However, this contribution to the total fluorescence is negligible in these experi ments. ${ }^{24,25}$

Polarized TIRF measurements were obtained $\sim 1 \mathrm{~h}$ after fluorescent labeled BSA was placed in contact with the quartz surface, to allow the BSA adsorption to reach quasiequilibrium. Measurements on bulk dissolved BSA were made with the sample in a conventional $1 \mathrm{~cm}^{2}$ rectangular quartz cuvette. All measurements, whether conventional or TIRF, were made in the steady state and taken in a SLM 4000 spectrofluorometer (SLM Instruments, Inc.; Urbana, Ill.) equiped with GlanThompson polarizers in the excitation and emission beams.

\section{Preparation of labeled BSA and the quartz surface}

The BSA protein molecules were covalently labeled with the fluorescent probes eosin-5-isothiocyanate (Molecular Probes, Inc.; Junction City, Or.), dansyl chloride (Molecular Probes), tetramethylrhodamine isothiocyanate (Research Organics, Inc.; Cleveland, Ohio), and 4-chloro-7-nitrobenzo-2-oxa-1, 2, diazol (Molecular Probes), as previously described. ${ }^{19}$ In all cases an average of five to ten probes per BSA molecule is attached. Preparation of the quartz surface is identical to that described previously. ${ }^{24}$

\section{RESULTS AND DISCUSSION}

The fluorescence polarization anisotropy of bulk dissolved and surface adsorbed fluorescently labeled BSA has been measured. The results of the measurements are summarized in Table $\mathrm{I}$. Calculation of the rotational diffusion constant $d_{3,3}$ from the anisotropy data for each fluorophore was performed using $\mathrm{Eq}$. (22) averaged over $\alpha_{1}^{0}$ and $\beta_{1}^{0}$. The Cartesian diffusion constants of the symmetrical carrier BSA at room temperature in phosphate buffered saline solution have been measured to be $D_{1,1}^{p f}=D_{2,2}^{\phi f}=8.3 \times 10^{5} \mathrm{~s}^{-1}$ and $D_{3,3}^{p f}=2.0$ $\times 10^{6} \mathrm{~s}^{-1}$ when bulk dissolved ${ }^{5,18}$ and are assumed to be $D_{1,1}^{\phi f}=D_{2,2}^{p f}=D_{3,3}^{p f}=0$ when surface adsorbed. The fluorescent lifetimes of the probes are noted in Table $I$.

Application of Eq. (22) requires knowledge of the four angles which determine the orientation of the absorption and emission dipole moments relative to the principal frame of the fluorophore. One of these angles is between the absorption and emission dipole, and is obtained from the anisotropy of randomly oriented, immobilized fluorophores. ${ }^{14}$ The others, which can be determined experimentally using time resolved anisotropy measurements, ${ }^{26}$ are assumed to be such that the absorption and emission dipoles both lie in a plane containing the axis of rotation of the fluorophore. This is a reasonable assumption given the planar shape of the fluorophores and the position of the reactive site for their covalent attachment to the carrier.

It is also assumed, as suggested previously, ${ }^{6}$ that one of the dipoles (absorption or emission) is oriented along the long axis of symmetry of the fluorophore's conjugated ring structure, which for these probes is $\sim 90^{\circ}$ from the axis of rotation. This assumption minimizes $d_{3,3}$; different choices of this dipole orientation angle increases $d_{3,3}$ by at most $10 \%$.

The calculated values of $d_{3,3}$ range widely for the fluorescent probes used in these experiments. For the bulk dissolved species $d_{3,3}$ ranges from a factor of $\sim 4$ 
(for dansyl) to $\sim 10^{3}$ (for eosin and rhodamine) larger than $D_{3,3}^{\phi f}$. The values of $d_{3,3}$ for the bulk dissolved compared to the surface adsorbed species for all cases, except dansyl, indicates that carrier adsorption significantly restricts the rotational mobility of the probe. This restricted rotational motion of the probe can be attributed to direct steric interference by the surface or to steric interference arising from a conformational change in the carrier.

The quantitative significance of the theory is tested by calculating $d_{3,3}$ from an expression of the anisotropy derived from a simple, approximate model of the luminophore and carrier system. In this simpler model the principal axis of the luminophore and carrier are identical [this is formally equivalent to assuming $D_{i, j}$ in Eq.

(1) to be diagonal and independent of luminophore orientation]. The anisotropy, for a symmetrical carrier, is then

$$
\begin{aligned}
r(t)= & \frac{r_{i}}{25}\left[1+2 \exp \left(-d_{3,3}^{\prime} t\right)\right. \\
& \left.+2 \exp \left(-4 d_{3,3}^{\prime} t\right)\right] \sum_{j=1}^{5} \exp \left(-E_{j} t\right)
\end{aligned}
$$

with

$$
\begin{aligned}
& E_{1}=E_{2}=5 D_{2,2}^{\phi f}+D_{1,1}^{\phi f}, \\
& E_{3}=6 D_{2,2}^{\phi f}, \quad E_{4}=E_{5}=2 D_{2,2}^{\phi f}+4 D_{1,1}^{\phi f}
\end{aligned}
$$

for $r_{i}$ the anisotropy of randomly oriented, immobilized luminophores. As shown in the table, application of Eq. (24) to the anisotropy data yields values for $d_{3,3}^{\prime}$ significantly greater than $d_{3,3}$ in every case. This would be expected intuitively since the correct theory allows depolarization of the emission from rotation of the luminophore out of the principal frame of the carrier.

Theory is developed here describing the rotational diffusive motion of a luminophore covalently attached to a large carrier also undergoing rotational diffusion. Application of this theory is described for fluorescently labeled BSA, when the BSA is in solution, and when it is adsorbed to a glass/buffer interface. The results indicate that different covalently bound fluorescent probes possess a wide range of rotational diffusion constants. The results also show that surface adsorption of the carrier may cause a significant reduction in the rotational mobility of the fluorophore.

The theory and experiments described in this paper determine the rotational diffusion constant of protein bound fluorophores. Knowledge of this parameter is useful in the application of other techniques such as singlet-singlet energy transfer, ${ }^{19}$ where the rapidity of the local motion of the donors and acceptors is essential to the interpretation of the spectroscopic data. ${ }^{27}$ The novel experimental technique of polarized TIRF extends the useful methods of polarized light spectroscopy to molecules adsorbed in equilibrium at a solid/liquid interface.

\section{ACKNOWLEDGMENTS}

The author acknowledges Sally Seidel for technical assistance and Nancy L. Thompson, Randy Stevenson, and Daniel Axelrod for helpful discussions. This work was supported by grants to Daniel Axelrod from the National Institutes of Health NS14565 and HL24039.

\section{APPENDIX}

Diagonalization of the $3 \times 3$ matrix $Q$ determines $E_{2, r}$ and $c_{i}^{\top}$, for $\tau=1,2,3, E_{2, \tau} ; \tau=1,2,3$ are roots of the cubic equation

$$
E_{2}^{3}+p E_{2}^{2}+q E_{2}+r=0,
$$

where

$$
\begin{aligned}
p= & -\left\{M_{0,0}+M_{1,1}+M_{2,2}-\sqrt{3 / 2} \exp \left(2 i \alpha_{1}^{0}\right) M_{2,0}\right\}, \quad \text { (A2) } \\
q= & {\left[M_{1,1}-\sqrt{3 / 2} \exp \left(2 i \alpha_{1}^{0}\right) M_{2,0}\right]\left[M_{0,0}+M_{2,2}\right]+M_{0,0} M_{2,2} } \\
& -2 \exp \left(2 i \alpha_{1}^{0}\right)\left[4 M_{1,0}^{2}-\exp \left(2 i \alpha_{1}^{0}\right) M_{2,0}^{2}\right], \\
r= & -\left[M_{1,1}-\sqrt{3 / 2} \exp \left(2 i \alpha_{1}^{0}\right) M_{2,0}\right]\left[M_{0,0} M_{2,2}\right. \\
& \left.-2 M_{2,0}^{2} \exp \left(4 i \alpha_{1}^{0}\right)\right]+2 M_{1,0}^{2} \exp \left(2 i \alpha_{1}^{0}\right) \\
& \times\left[3 M_{0,0}+M_{2,2}-2 \sqrt{6} \exp \left(2 i \alpha_{1}^{0}\right) M_{2,0}\right] .
\end{aligned}
$$

$E_{2, \tau}$ is given by ${ }^{28}$

$$
E_{2, \tau}= \begin{cases}A+B & ; \tau=1 \\ -\frac{1}{2}(A+B)+\frac{1}{2}(A-B) \sqrt{-3} ; & \tau=2 \\ -\frac{1}{2}(A+B)-\frac{1}{2}(A-B) \sqrt{-3} ; & \tau=3,\end{cases}
$$

where

$$
\begin{aligned}
& A=\left(-\frac{b}{2}+\sqrt{\frac{b^{2}}{4}+\frac{a^{3}}{27}}\right)^{1 / 3}, \\
& B=\left(-\frac{b}{2}-\sqrt{\frac{b^{2}}{4}+\frac{a^{3}}{27}}\right)^{1 / 3},
\end{aligned}
$$

and

$$
a=\frac{1}{3}\left(3 q-p^{2}\right), \quad b=\frac{1}{27}\left(2 p^{3}-9 p q+27 r\right) .
$$

The diagonalization of $\mathbf{Q}$ also implies

$$
\begin{aligned}
& c_{0}^{\tau}=\frac{x_{\tau}}{\left(1+x_{\tau}^{2}+y_{\tau}^{2}\right)^{1 / 2}}, \\
& c_{1}^{\tau}=\frac{-y_{\tau}}{\left(1+x_{\tau}^{2}+y_{\tau}^{2}\right)^{1 / 2}}, \quad c_{2}^{\top}=\frac{1}{\left(1+x_{\tau}^{2}+y_{\tau}^{2}\right)^{1 / 2}},
\end{aligned}
$$

and consequently $b_{0}^{\tau}=c_{0}^{\tau}, \quad b_{ \pm 1}^{\tau}= \pm \sqrt{1 / 2} c_{1}^{\tau} \exp \left(\mp i \alpha_{1}^{0}\right), \quad b_{ \pm 2}^{\tau}$ $=\sqrt{1 / 2} c_{2}^{\tau} \exp \left(\mp 2 i \alpha_{1}^{0}\right)$ given that

$$
\begin{aligned}
x_{\tau}= & \frac{\sqrt{6} \exp \left(2 i \alpha_{1}^{0}\right) M_{2,0}-M_{2,2}+E_{2, \tau}}{\sqrt{2} M_{2,0} \exp \left(2 i \alpha_{1}^{0}\right)-\sqrt{3}\left(M_{0,0}-E_{2, \tau}\right)}, \\
y_{\tau}= & \frac{1}{\sqrt{2} \exp \left(i \alpha_{1}^{0}\right) M_{1,0}} \\
& \times\left\{\frac{\left[M_{0,0}-E_{2, I}\right]\left[E_{2, \tau}-M_{2,2}\right]+2 \exp \left(4 i \alpha_{1}^{0}\right) M_{2,0}^{2}}{\sqrt{2} M_{2,0} \exp \left(2 i \alpha_{1}^{0}\right)-\sqrt{3}\left(M_{0,0}-E_{2, \tau}\right)}\right\} .
\end{aligned}
$$

Diagonalization of $R$ implies, for $\tau=4,5$ :

$$
\begin{aligned}
E_{2,5} & =\frac{1}{2}\left[M_{1,1}+M_{2,2}+\sqrt{3 / 2} \exp \left(2 i \alpha_{1}^{0}\right) M_{2,0} \pm\left(\left[M_{2,2}-M_{1,1}\right.\right.\right. \\
& \left.\left.\left.-\sqrt{3 / 2} \exp \left(2 i \alpha_{1}^{0}\right) M_{2,0}\right]^{2}+24 \exp \left(2 i \alpha_{1}^{0}\right) M_{1,0}^{2}\right)^{1 / 2}\right]
\end{aligned}
$$

and $c_{i}^{\top}$ :

$$
c_{0}^{\top}=0 ; \quad c_{1}^{\top}=\frac{E_{2, \tau}-M_{2,2}}{\left[6 \exp \left(2 i \alpha_{1}^{0}\right) M_{1,0}^{2}+\left(E_{2, \tau}-M_{2,2}\right)^{2}\right]^{1 / 2}} ;
$$




$$
c_{2}^{\top}=\frac{\sqrt{6} \exp \left(i \alpha_{1}^{0}\right) M_{1, \ell}}{\left[6 \exp \left(2 i \alpha_{1}^{0}\right) M_{1,0}^{2}+\left(E_{2},-M_{2,2}\right)^{2}\right]^{1 / 2}},
$$

consequently, $b_{0}^{\tau}=0, b_{ \pm 1}^{\tau}=\sqrt{1 / 2} c_{1}^{\tau} \exp \left(\mp i \alpha_{1}^{0}\right), b_{ \pm 2}^{\tau}$ $= \pm \sqrt{1 / 2} c_{2}^{\top} \exp \left(\mp 2 i \alpha_{1}^{0}\right)$.

${ }^{1}$ R. Rigler and M. Ehrenberg, Q. Rev. Biophys. 6, 139 (1973).

${ }^{2}$ D. C. Yang, W. E. Gall, and G. M. Edelman, J. Biol. Chem. 249(21), 7018 (1974).

${ }^{3} \mathrm{~J}$. Yguerabide, H. F. Epstein, and L. Stryer, J. Mol. Biol. $51,573(1970)$.

${ }^{4} \mathrm{~J}$. R. Lakowicz and G. Weber, Biophys. J. 32, 591 (1980).

${ }^{5}$ W. F. Harrington, P. Johnson, and R. H. Ottewill, Biochem. J. 62, 562 (1956).

${ }^{6}$ P. Wahl and G. Weber, J. Mol. Biol. 30, 371 (1967).

${ }^{7}$ G. Lipari and A. Szabo, Biophys. J. 30, 489 (1980).

${ }^{8}$ I. Munro, I. Pecht, and L. Stryer, Proc. Natl. Acad. Sci. 76, 56 (1979).

${ }^{9}$ F. Jahnig, Proc. Natl. Acad. Sci. 76, 6361 (1979).

${ }^{10} \mathrm{~K}$. Kinosita, Jr., S. Kawato, and A. Ikegami, Biophys. J. 20, 289 (1977).

${ }^{11}$ M. Shinitzky and P. Henkart, Int. Rev. Cytol. 60, 121 (1979).

${ }^{12}$ R. J. Cherry, FEBS Lett. 55, 1 (1975).
${ }^{13}$ R. H. Austin, S. S. Chan, and T. M. Jovin, Proc. Natl. Acad. Sci. 76(11), 5650 (1979).

${ }^{14}$ D. Axelrod, Biophys. J. 26, 557 (1979).

${ }^{15} \mathrm{M}$. Shinitzky and Y. Barenholz, Biochim. Biophys. Acta 515, 367 (1978).

${ }^{16} \mathrm{Y}$. Gottlieb and P. Wahl, J. Chim. Phys. 60, 849 (1963).

${ }^{17}$ J. M. Schurr, Chem. Phys. 65, 417 (1982).

${ }^{18} \mathrm{~S}$. Krause and C. T. O'Konski, J. Am. Chem. Soc. 81 , $5082(1959)$.

${ }^{19}$ T. P. Burghardt and D. Axelrod, Biochemistry 22, 979 (1983).

${ }^{20}$ L. D. Favro, Phys. Rev. 119, 53 (1960).

${ }^{21}$ L. D. Landau and E. M. Lifshitz, Quantum Mechanics: Nonrelativistic Theory (Pergamon, New York, 1975).

${ }^{22}$ A. S. Davydov, Quantum Mechanics (NEO, Ann Arbor, Michigan, 1966).

${ }^{23}$ N. J. Harrick, Internal Reflection Spectroscopy (Wiley, New York, 1966).

${ }^{24}$ T. P. Burghardt and D. Axelrod, Biophys. J. 33, 455 (1981).

${ }^{25} \mathrm{~N}$. L. Thompson, Ph.D. thesis, University of Michigan, 1982.

${ }^{26}$ M. D. Barkley, A. A. Kowalczyk, and L. Brand, J. Chem. Phys. 75(7), 3581 (1981).

${ }^{27}$ R. E. Dale and J. Eisinger, Biopolymers 13, 1573 (1974).

${ }^{28}$ CRC Standard Mathematical Tables, 16 th ed., edited by S. M. Selby (Chemical Rubber, Clevland, Ohio, 1968).

${ }^{29} \mathrm{R}$. Haugland (private communication).

${ }^{30}$ H. E. Lessing and A. Von Jena, Chem. Phys. 41, 395 (1979). 